\title{
The finite temperature QCD phase transition with domain wall fermions.
}

\author{
Pavlos M. Vranas * a

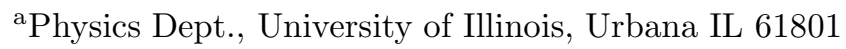

Results from the Columbia lattice group study of the QCD finite temperature phase transition with dynamical domain wall fermions on $16^{3} \times 4$ lattices are presented. These results include an investigation of the $U(1)$ axial symmetry breaking above but close to the transition, the use of zero temperature calculations that set the scale at the transition and preliminary measurements close to the transition.

\section{Introduction}

Domain wall fermions (DWF) [1] provide a way to separate the approach to the continuum limit from the approach to the chiral limit. At fixed lattice spacing the chiral limit is approached as the size $L_{s}$ of the "fifth" direction is increased. Furthermore, since this regulator does not break flavor symmetry and since the computing cost is only linear in $L_{s}$ it may be possible to simulate two flavor QCD with three light pions close to the finite temperature transition using today's supercomputers.

Preliminary results of QCD thermodynamic studies with DWF were presented in [2]. Results from simulations on $8^{3} \times 4$ lattices indicated the presence of a low temperature phase with spontaneous chiral symmetry breaking but intact flavor symmetry and a high temperature phase with the full $S U(2) \times S U(2)$ chiral flavor symmetry. Also, preliminary results on $16^{3} \times 4$ lattices just above the deconfining transition indicated a difference in the $\pi$ and $\delta$ susceptibilities and screening masses. This was preliminary evidence for the anomalous breaking of the $U_{A}(1)$ symmetry above the transition.

Here the above preliminary results are extended in two directions: 1) The investigation of the $U_{A}(1)$ axial symmetry breaking is completed with results at two values of the coupling close to the transition. 2) A full study of the transi-

\footnotetext{
*In collaboration with P. Chen, N. Christ, G. Fleming, A. Kaehler, T. Klassen, C. Malureanu, R. Mawhinney, G. Siegert C. Sui, L. Wu, Y. Zhestkov. Supported in part by DOE grant \# DE-FG02-92ER40699 and in part by NSF grant \# NSF-PHY96-05199 (PMV).
}

tion region on $16^{3} \times 4$ lattices is performed and is accompanied by scale setting calculations at the transition point to determine the transition temperature and the pion mass. Some of these results have already been reported in [3]. Also see [4,5].

The DWF action used in this work is as in [6] with the modifications as in [7]. Some details on the numerical methods can be found in [2]. For a review on DWF see [8] and references therein.

\section{The $U_{A}(1)$ symmetry above $T_{c}$}

An important question concerns the role of the $U_{A}(1)$ anomaly above the finite temperature QCD transition. Earlier attempts to address this question [9,10] using staggered fermions could not produce conclusive results because staggered fermions break the $U_{A}(1)$ symmetry already at the classical level. On the other hand, DWF bring this problem under control. In particular at $L_{s}=\infty$ massless DWF do not break the $U_{A}(1)$ symmetry at the classical level. Also, the DWF Dirac operator can have exact zero modes [11, the necessary property for anomalous breaking. In 12] it was shown that this property is maintained to a good approximation for fairly small masses and for $L_{s}$ as small as $\approx 10$.

The difference of the susceptibilities $\chi$ of the $\pi$ and $\delta$ is used as a measure of anomalous symmetry breaking. This difference was measured as a function of the bare quark mass $m_{f}$ on a $16 \times 4$ lattice at $\beta=5.45$ and $\beta=5.40\left(\beta_{c} \approx 5.325\right)$. The results are presented in figure 1 . The lines are fits to $c_{0}+c_{2} m_{f}^{2}$ and have $\chi^{2} /$ d.o.f. $\approx 1$. The absence of a linear term indicates that for $L_{s}=16$ the chi- 
ral symmetry is effectively restored. The $m_{f}=0$ extrapolated values are $0.26(6)$ at $\beta=5.40$ and $0.08(3)$ at $\beta=5.45$. Although both are not zero by a statistically significant amount their value is small when compared with $\chi_{\pi}$ and $\chi_{\delta}$ which are $\approx 8$. Universality arguments require that if the QCD phase transition is to be second order, the anomalous $U(1)_{A}$ must be broken. It is an open question as to whether the small size of the $U(1)_{A}$ symmetry breaking seen here is sufficient to support this theoretical prediction that the two-flavor QCD phase transition is second order [13]. For quenched QCD thermodynamic studies with DWF see 14, 15, 17, and with overlap fermions see 16.

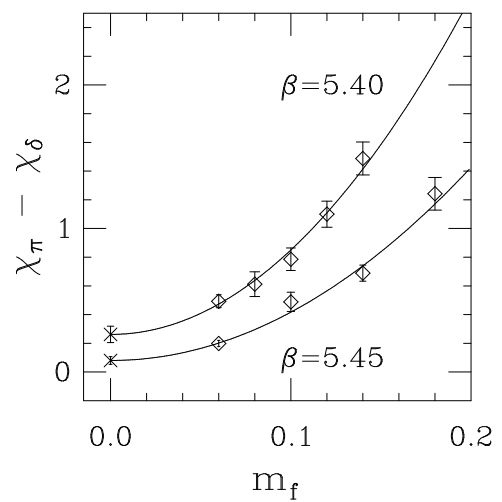

Figure 1. $16^{4} \times 4, L_{s}=16$ and $m_{0}=1.9$. The fits are to $c_{0}+c_{2} m_{f}^{2}$ and the stars are the $m_{f}=0$ extrapolated values.

\section{The transition}

The transition region $(5.2<\beta<5.4)$ is studied on $16^{3} \times 4$ lattices using $m_{f}=0.02$, a domain wall height $m_{0}=1.9$ and $L_{s}=24$. These values were chosen based on previous exploratory studies on smaller lattices 22,3]. In particular $m_{0}=1.9$ was found to produce two flavor physics and $L_{s}=24$ to be large enough to keep the residual chiral symmetry breaking effects on the chiral condensate to the few percent level above the transition and to $\approx 15 \%$ level below the transition. The results are shown in figure 2. The gauge part of the action is a standard Wilson plaquette action.

In order to set the scale a simulation [20] on an $8^{3} \times 32$ lattice was done at $\beta=5.325$ corre- sponding to the middle of the transition region . It was found that in lattice units $m_{\rho}=1.18(3)$ and $m_{\pi}=0.654(3)$. This gives a value for the critical temperature $T_{c}=163(4) \mathrm{MeV}$ and $m_{\pi}=427(11) \mathrm{MeV}$. The critical temperature is in agreement with results obtained from other fermion regulators [5]. The pion mass is clearly too heavy to be able to extract useful information regarding the order of the transition. More sophisticated studies [18] indicate that the residual chiral symmetry breaking effects are much larger than expected and in order to obtain a physical pion mass $L_{s} \approx 100$ may be needed.

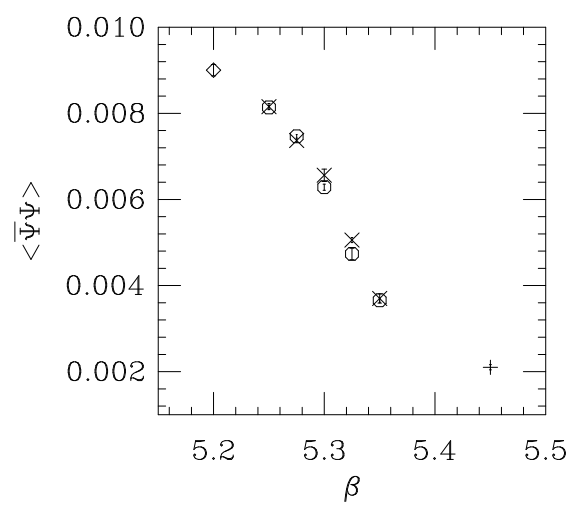

Figure 2. $\langle\bar{\psi} \psi\rangle$ vs. $\beta$ with $m_{0}=1.9, m_{f}=0.02$ and $L_{s}=24$. The circles correspond to $16^{3} \times 4$ lattice with an ordered initial configuration (ic), the crosses to a $16^{3} \times 4$ with disordered ic, the diamond to an $8^{3} \times 4$ with ordered ic, and the cross to an $8^{3} \times 4$ with disordered ic. The gauge part of the action is a Wilson plaquette action.

Large residual chiral symmetry breaking effects have been related to the "roughness" of the gauge field configurations 11,6, 7, 7, 19. Therefore a possible "cure" could be to use improved gauge actions. This idea was explored in zero temperature quenched QCD by using an Iwasaki improved gauge action. Dramatic improvements were seen at couplings that correspond to $N_{t}=4$ finite temperature quenched QCD just above the transition [20]. These results prompted a study of the dynamical QCD finite temperature transition using Iwasaki improved gauge action with $c_{1}=-0.331$. The results are presented in figure 3 . All parameters are the same as in figure 2 . 


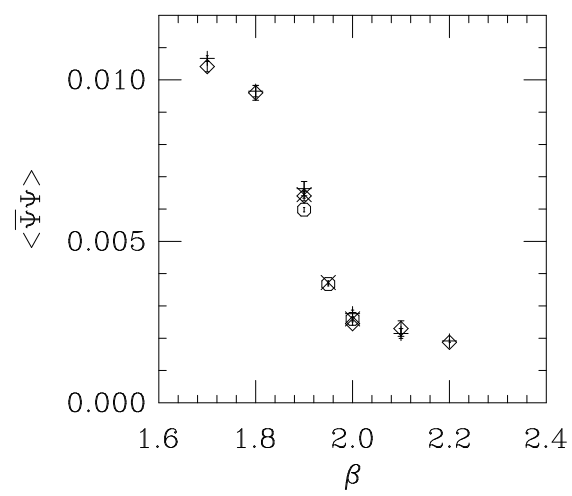

Figure 3. Same as in figure 2 but with an Iwasaki improved gauge action with $c_{1}=-0.331$.

Again, in order to set the scale a simulation 20] on an $8^{3} \times 32$ lattice was done at $\beta=1.9$ corresponding to the middle of the transition region. It was found that in lattice units $m_{\rho}=1.163(21)$ and $m_{\pi}=0.604(3)$. This gives a value for the critical temperature $T_{c}=166(3) \mathrm{MeV}$ and $m_{\pi}=400(7) \mathrm{MeV}$. The critical temperature is in agreement with the Wilson plaquette action results. However, the pion mass did not get lighter by a significant amount.

\section{Conclusions}

The difference of the susceptibilities of the $\pi$ and $\delta$ was measured at two couplings just above the finite temperature transition. This difference was found to be non-zero by a statistically significant amount indicating that $U_{A}(1)$ remains broken above but close to the transition. However, the value of the difference of the susceptibilities is much smaller than the susceptibilities themselves (by a factor of $\approx 30-100$ for each point) suggesting that the size of $U_{A}(1)$ is indeed rather small at these couplings. It is an open question as to what effect this may have to the order of the transition.

The transition region was studied by dynamical simulations on $16^{3} \times 4$ lattices with $L_{s}=24$ and the critical coupling was located. At that coupling zero temperature simulations were done on $8^{3} \times 32$ lattices in order to set the scale. For the gauge part of the action two different types were used. A standard plaquette action gave critical temperature $T_{c}=163(4) \mathrm{MeV}$ and $m_{\pi}=427(11) \mathrm{MeV}$. In an effort to reduce the pion mass an Iwasaki gauge action was also used. For that action $T_{c}=166(3) \mathrm{MeV}$ and $m_{\pi}=400(7) \mathrm{MeV}$. Unfortunately the pion mass is still too heavy to allow a study of the order of the transition. Larger values of $L_{s}$ or alternative improvement techniques must be employed in order to reduce the pion mass to physical values and still be able to study the transition with the available computing resources.

All calculations were done on the 400 Gflops QCDSP machine at Columbia University.

\section{REFERENCES}

1. D.B. Kaplan, Phys. Lett. B288 (1992) 342.

2. P. Chen et.al., Nucl. Phys. B73 (Proc. Suppl.) (1999) 456.

3. P. Vranas, DPF 99 proc., hep-lat/9903024.

4. R. Mawhinney, these proceedings.

5. F. Karsch, these proc., hep-lat/9909006.

6. V. Furman, Y. Shamir, Nucl. Phys. B439 (1995) 54.

7. P.M. Vranas, Phys. Rev. D57 (1998) 1415.

8. T. Blum, Nucl. Phys. B73 (Proc. Suppl.) (1999) 167.

9. S. Chandrasekharan et.al., Phys. Rev. Lett. 82 (1999) 2463.

10. J.B. Kogut et.al., Phys. Rev. D58 (1998) 054504 .

11. R. Narayanan, H. Neuberger, Nucl. Phys. B443 (1995) 305.

12. P. Chen et.al., Phys. Rev. D59 (1999) 054508.

13. R. Pizarski and F. Wilczek, Phys. Rev. D29 (1984) 338.

14. P. Chen et.al., Nucl. Phys. B73 (Proc. Suppl.) (1999) 207.

15. P. Chen et.al., Nucl. Phys. B73 (Proc. Suppl.) (1999) 405.

16. U. Heller, these proc., hep-lat/9908036.

17. D. Sinclair, these proceedings.

18. G. Fleming, these proceedings.

19. U. Heller et.al., Nucl. Phys. B535 (1998) 403.

20. L. Wu, these proceedings. 